\title{
Publisher's Note: Detectable MeV neutrinos from black hole neutrino-dominated accretion flows [Phys. Rev. D 93, 123004 (2016)]
}

\author{
Tong Liu@, Bing Zhang, Ye Li, Ren-Yi Ma, and Li Xue
}

Q (Received 2 November 2020; published 18 November 2020)

DOI: $10.1103 /$ PhysRevD.102.109902

This paper was published online on 9 June 2016 with omissions of a corresponding author footnote label and byline footnote indicator for the fourth author. The footnote for the corresponding author should read as " Corresponding author: tongliu@xmu.edu.cn" and the footnote for Ma should read as "“ryma@xmu.edu.cn”. The paper has been corrected as of 2 November 2020. The paper is incorrect in the printed version of the journal. 